\title{
An analysis of the user occupational class through Twitter content
}

\author{
Daniel Preoţiuc-Pietro ${ }^{1}$, Vasileios Lampos² ${ }^{2}$ and Nikolaos Aletras ${ }^{2}$ \\ ${ }^{1}$ Computer \& Information Science, University of Pennsylvania \\ 2 Department of Computer Science, University College London \\ danielpresas.upenn.edu, \{v.lampos,n.aletras\}@ucl.ac.uk
}

\begin{abstract}
Social media content can be used as a complementary source to the traditional methods for extracting and studying collective social attributes. This study focuses on the prediction of the occupational class for a public user profile. Our analysis is conducted on a new annotated corpus of Twitter users, their respective job titles, posted textual content and platform-related attributes. We frame our task as classification using latent feature representations such as word clusters and embeddings. The employed linear and, especially, non-linear methods can predict a user's occupational class with strong accuracy for the coarsest level of a standard occupation taxonomy which includes nine classes. Combined with a qualitative assessment, the derived results confirm the feasibility of our approach in inferring a new user attribute that can be embedded in a multitude of downstream applications.
\end{abstract}

\section{Introduction}

The growth of online social networks provides the opportunity to analyse user text in a broader context (Tumasjan et al., 2010; Bollen et al., 2011; Lampos and Cristianini, 2012). This includes the social network (Sadilek et al., 2012), spatio-temporal information (Lampos and Cristianini, 2010) and personal attributes (Al Zamal et al., 2012). Previous research has analysed language differences in user attributes like location (Cheng et al., 2010), gender (Burger et al., 2011), impact (Lampos et al., 2014) and age (Rao et al., 2010), showing that language use is influenced by them. Therefore, user text allows us to infer these properties. This user profiling is important not only for sociolinguistic studies, but also for other applications: recommender systems to provide targeted advertising, analysts who study different opinions in each social class or integration in text regression tasks such as voting intention (Lampos et al., 2013).

Social status reflected through a person's occupation is a factor which influences language use (Bernstein, 1960; Bernstein, 2003; Labov, 2006). Therefore, our hypothesis is that language use in social media can be indicative of a user's occupational class. For example, executives may write more frequently about business or financial news, while people in manufacturing positions could refer more to their personal interests and less to job related activities. Similarly, we expect some categories of people, like those working in sales and customer services, to be more social or to use more informal language.

Focusing on the microblogging platform of Twitter, we explore our hypothesis by studying the task of predicting a user's occupational class given platform-related attributes and generated content, i.e. tweets. That has direct applicability in a broad range of areas from sociological studies, which analyse the behaviour of different occupations, to recruiting companies that target people for new job opportunities. For this study, we created a publicly available data set of users, including their profile information and historical text content as well as a label to an occupational class from the "Standard Occupational Classification" taxonomy (see Section 2).

We frame our task as classification, aiming to identify the most likely job class for a given user based on profile and a variety of textual features: general word embeddings and clusters (or 'topics'). Both linear and non-linear classification methods are applied with a focus on those that can assist interpretation and offer qualitative insights. We find that text features, especially word clusters, lead to good predictive performance. Accuracy for our best model is well above $50 \%$ for 9-way classifi- 
cation, outperforming competitive methods. The best results are obtained using the Bayesian nonparametric framework of Gaussian Processes (Rasmussen and Williams, 2006), which also accommodates feature interpretation via the Automatic Relevance Determination. This allows us to get insight into differences in language use across job classes and, finally, assess our original hypothesis about the thematic divergence across them.

\section{Standard Occupational Classification}

To enable the user occupation study, we adopt a standardised job classification taxonomy for mapping Twitter users to occupations. The Standard Occupational Classification (SOC) ${ }^{1}$ is a UK government system developed by the Office of National Statistics for classifying occupations. Jobs are categorised hierarchically based on skill requirements and content. The SOC scheme includes nine major groups coded with a digit from 1 to 9 . Each major group is divided into sub-major groups coded with 2 digits, where the first digit indicates the major group. Each sub-major group is further divided into minor groups coded with 3 digits and finally, minor groups are divided into unit groups, coded with 4 digits. The unit groups are the leaves of the hierarchy and represent specific jobs related to the group.

Table 1 shows a part of the SOC hierarchy. In total, there are 9 major groups, 25 sub-major groups, 90 minor groups and 369 unit groups. Although other hierarchies exist, we use the SOC because it has been published recently (in 2010), includes newly introduced jobs, has a balanced hierarchy and offers a wide variety of job titles that were crucial in our data set creation.

\section{Data}

To the best of our knowledge there are no publicly available data sets suitable for the task we aim to investigate. Thus, we have created a new one consisting of Twitter users mapped to their occupation, together with their profile information and historical tweets. We use the account's profile information to capture users with self-disclosed occupations. The potential self-selection bias is acknowledged, but filtering content via self disclosure

\footnotetext{
${ }^{1}$ http: //www.ons.gov.uk/ons / guide-method/classifications/ current-standard-classifications/ soc2010/index. html; accessed on 24/02/2015.
}

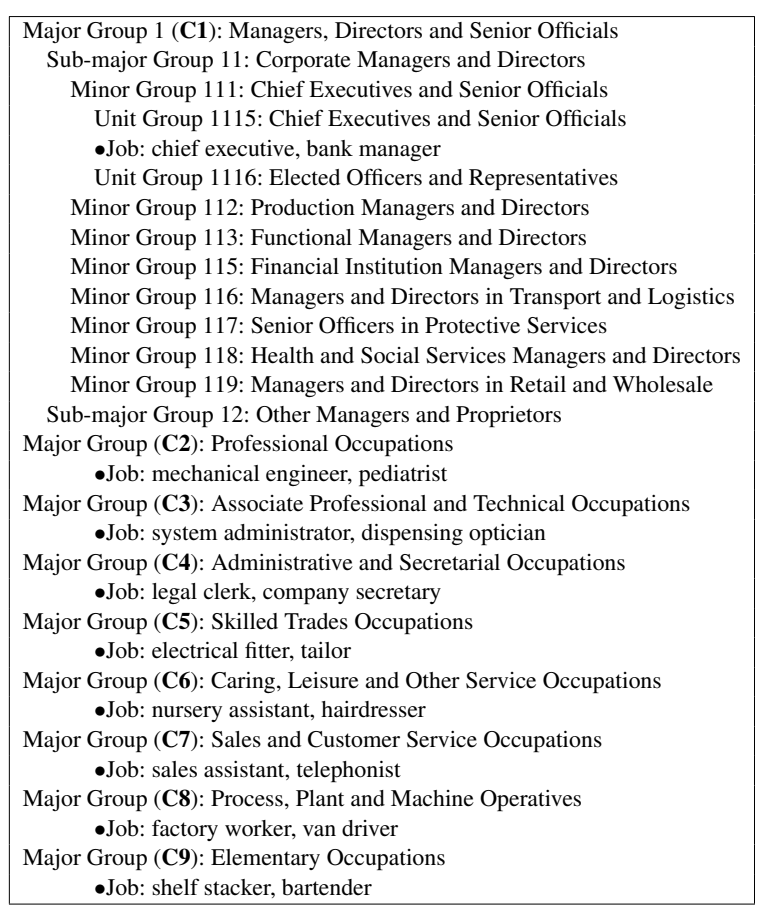

Table 1: Subset of the SOC classification hierarchy.

is widespread when extracting large-scale data for user attribute inference (Pennacchiotti and Popescu, 2011; Coppersmith et al., 2014).

Similarly to Hecht et al. (2011), we first assess the proportion of Twitter accounts with a clear mention to their occupation by annotating the user description field of a random set of 500 users. There were chosen from the random $1 \%$ sample, having at least 200 tweets in their history and with a majority of English tweets. There, we can identify the following categories: no description (12.2\%), random information $(22 \%)$, user information but not occupation related (45.8\%), and job related information (20\%).

To create our data set, we thus use the user description field to search for self-disclosed job titles provided by the 4-digit SOC unit groups, since they contain specific job titles. We queried Twitter's Search API to retrieve for each job title a maximum of 200 accounts which best matched occupation keywords. Then, we aggregated the accounts into the 3-digit (minor) categories. To remove potential ambiguity in the retrieved set, we manually inspected accounts in each minor category and filtered out those that belong to companies, contain no description or the description provided does not indicate that the user has a job corresponding to the minor category. In total, around $50 \%$ of the accounts were removed by manual inspection per- 
formed by the authors. We also removed users in multiple categories and or users that have tweeted less than 50 times in their history. Finally, we eliminated all 3-digit categories that contained less than 45 user accounts after this filtering. This process produced a total number of 5,191 users from $55 \mathrm{mi}$ nor groups (22 sub-major groups), spread across all nine major SOC groups. The distribution of users across these nine groups is: $9.7 \%, 34.5 \%, 20.6 \%$, $3.8 \%, 16.7 \%, 6.1 \%, 1.4 \%, 4.2 \%$, and $3 \%$ (following the ordering of Table 1). In our data set the most well represented minor occupational groups are 'Functional Managers and Directors' (184 users - code 113), 'Therapy Professionals' (159 users code 222) and 'Quality and Regulatory Professionals' (158 users - code 246), whereas the least represented ones are 'Textile and Garment Trades' (45 users - code 541), 'Elementary Security Occupations' (46 users - code 924), 'Elementary Cleaning Occupations' (47 users - code 923). The mean number of users in the minor classes is equal to 94.4 with a standard deviation of 35.6. For these users, we have collected all their tweets, going as far back as the latest 3,200, and their profile information. The final data set consists of $10,796,836$ tweets collected around 5 August 2014 and is openly available. $^{2}$

A separate Twitter data set is used as a reference corpus in order to build the feature representations detailed in Section 4. This data set is an extract from the Twitter Gardenhose stream (a 10\% representative sample of the entire Twitter stream) from 2 January to 28 February 2011. Based on this content, we also build the vocabulary for the text features, containing the most frequent 71,555 words. We tokenise and filter for English using the Trendminer preprocessing pipeline (Preoţiuc-Pietro et al., 2012).

\section{Features}

In this section, we overview the features used in the occupational class prediction task. They are divided into two types: (1) user level features, (2) textual features.

\subsection{User Level Features (UserLevel)}

The user level features are based on the general user information or aggregated statistics about the tweets. Table 2 introduces the 18 features in this

\footnotetext{
${ }^{2}$ http://www.sas.upenn.edu/ danielpr/ jobs.tar.gz
}

\begin{tabular}{|c|c|}
\hline$u_{1}$ & number of followers \\
$u_{2}$ & number of friends \\
$u_{3}$ & number of times listed \\
$u_{4}$ & follower/friend ratio \\
$u_{5}$ & proportion of non-duplicate tweets \\
$u_{6}$ & proportion of retweeted tweets \\
$u_{7}$ & average no. of retweets/tweet \\
$u_{8}$ & proportion of retweets done \\
$u_{9}$ & proportion of hashtags \\
$u_{10}$ & proportion of tweets with hashtags \\
$u_{11}$ & proportion of tweets with @-mentions \\
$u_{12}$ & proportion of @-replies \\
$u_{13}$ & no. of unique @-mentions in tweets \\
$u_{14}$ & proportion of tweets with links \\
$u_{15}$ & no. of favourites the account made \\
$u_{16}$ & avg. number of tweets/day \\
$u_{17}$ & total number of tweets \\
$u_{18}$ & proportion of tweets in English \\
\hline
\end{tabular}

Table 2: User level attributes for a Twitter user.

category.

\subsection{Textual Features}

The textual features are derived from the aggregated set of user's tweets. We use our reference corpus to represent each user as a distribution over these features. We ignore the bio field from building textual features to avoid introducing biases from our data collection method. While this is a restriction, our analysis showed that in less than $20 \%$ of the cases the information in the bio is directly relevant to the occupation.

\subsubsection{SVD Word Embeddings (SVD-E)}

We use a more abstract representation of words than simple unigram counts in order to aid interpretability of our analysis. We compute a word to word similarity matrix from our reference corpus. Normalised Pointwise Mutual Information (NPMI) (Bouma, 2009) is used to compute word to word similarity. NPMI is an information theoretic measure indicating which words co-occur in the same context, where the context is represented by a whole tweet:

$$
\operatorname{NPMI}(x, y)=-\log \mathrm{P}(x, y) \cdot \log \frac{\mathrm{P}(x, y)}{\mathrm{P}(x) \cdot \mathrm{P}(y)} .
$$

We then perform singular value decomposition (SVD) on the word to word similarity matrix and obtain an embedding of words into a low dimensional space. In our experiments we tried the following dimensionalities: 30, 50, 100 and 200. The feature representation for each user is obtained summing over each of the embedding dimensions across all words. 


\subsubsection{NPMI Clusters (SVD-C)}

We use the NPMI matrix described in the previous paragraph to create hard clusters of words. These clusters can be thought as 'topics', i.e. words that are semantically similar. From a variety of clustering techniques we choose spectral clustering (Shi and Malik, 2000; Ng et al., 2002), a hard-clustering approach which deals well with high-dimensional and non-convex data (von Luxburg, 2007). Spectral clustering is based on applying SVD to the graph Laplacian and aims to perform an optimal graph partitioning on the NPMI similarity matrix. The number of clusters needs to be pre-specified. We use 30, 50, 100 and 200 clusters - numbers were chosen a priori based on previous work (Lampos et al., 2014). The feature representation is the standardised number of words from each cluster.

Although there is a loss of information compared to the original representation, the clusters are very useful in the model analysis step. Embeddings are hard to interpret because each dimension is an abstract notion, while the clusters can be interpreted by presenting a list of the most frequent or representative words. The latter are identified using the following centrality metric:

$$
\mathrm{C}_{w}=\frac{\sum_{x \in c} \operatorname{NPMI}(w, x)}{|c|-1}
$$

where $c$ denotes the cluster and $w$ the target word.

\subsubsection{Neural Embeddings (W2V-E)}

Recently, there has been a growing interest in neural language models, where the words are projected into a lower dimensional dense vector space via a hidden layer (Mikolov et al., 2013b). These models showed they can provide a better representation of words compared to traditional language models (Mikolov et al., 2013c) because they capture syntactic information rather than just bag-of-context, handling non-linear transformations. In this low dimensional vector space, words with a small distance are considered semantically similar. We use the skipgram model with negative sampling (Mikolov et al., 2013a) to learn word embeddings on the Twitter reference corpus. In that case, the skip-gram model is factorising a word-context PMI matrix (Levy and Goldberg, 2014). We use a layer size of 50 and the Gensim implementation. ${ }^{3}$

\footnotetext{
${ }^{3}$ http: / / radimrehurek. com/gensim/ models/word2vec.html
}

\subsubsection{Neural Clusters (W2V-C)}

Similar to the NPMI cluster, we use the neural embeddings in order to obtain clusters of related words, i.e. 'topics'. We derive a word to word similarity matrix using cosine similarity on the neural embeddings. We apply spectral clustering on this matrix to obtain 30, 50, 100 and 200 word clusters.

\section{Classification with Gaussian Processes}

In this section, we briefly overview Gaussian Process (GP) for classification, highlighting our motivation for using this method. GPs formulate a Bayesian non-parametric machine learning framework which defines a prior on functions (Rasmussen and Williams, 2006). The properties of the functions are given by a kernel which models the covariance in the response values as a function of its inputs. Although GPs form a powerful learning tool, they have only recently been used in NLP research (Cohn and Specia, 2013; Preoţiuc-Pietro and Cohn, 2013) with classification applications limited to (Polajnar et al., 2011).

Formally, GP methods aim to learn a function $f: \mathbb{R}^{d} \rightarrow \mathbb{R}$ drawn from a GP prior given the inputs $\boldsymbol{x} \in \mathbb{R}^{d}$ :

$$
f(\boldsymbol{x}) \sim \mathcal{G P}\left(m(\boldsymbol{x}), k\left(\boldsymbol{x}, \boldsymbol{x}^{\prime}\right)\right),
$$

where $m(\cdot)$ is the mean function (here 0 ) and $k(\cdot, \cdot)$ is the covariance kernel. Usually, the Squared Exponential (SE) kernel (a.k.a. RBF or Gaussian) is used to encourage smooth functions. For the multidimensional pair of inputs $\left(\boldsymbol{x}, \boldsymbol{x}^{\prime}\right)$, this is:

$$
k_{\operatorname{ard}}\left(\boldsymbol{x}, \boldsymbol{x}^{\prime}\right)=\sigma^{2} \exp \left[\sum_{i}^{d}-\frac{\left(x_{i}-x_{i}^{\prime}\right)^{2}}{2 l_{i}^{2}}\right] \text {, }
$$

where $l_{i}$ are lengthscale parameters learnt only using training data by performing gradient ascent on the type-II marginal likelihood. Intuitively, the lengthscale parameter $l_{i}$ controls the variation along the $i$ input dimension, i.e. a low value makes the output very sensitive to input data, thus making that input more useful for the prediction. If the lengthscales are learnt separately for each input dimension the kernel is named SE with Automatic Relevance Determination (ARD) (Neal, 1996).

Binary classification using GPs 'squashes' the real valued latent function $f(x)$ output through a logistic function: $\pi(\boldsymbol{x}) \triangleq \mathrm{P}(y=1 \mid \boldsymbol{x})=\sigma(f(\boldsymbol{x}))$ in a similar way to logistic regression classification. The object of the GP inference is the distribution 
of the latent variable corresponding to a test case $x_{*}$ :

$$
\mathrm{P}\left(f_{*} \mid \boldsymbol{x}, \boldsymbol{y}, x_{*}\right)=\int \mathrm{P}\left(f_{*} \mid \boldsymbol{x}, x_{*}, f\right) \mathrm{P}(f \mid \boldsymbol{x}, \boldsymbol{y}) d f,
$$

where $\mathrm{P}(f \mid \boldsymbol{x}, \boldsymbol{y})=\mathrm{P}(\boldsymbol{y} \mid f) \mathrm{P}(f \mid \boldsymbol{x}) / \mathrm{P}(\boldsymbol{y} \mid \boldsymbol{x})$ is the posterior over the latent variables. If the likelihood $\mathrm{P}(\boldsymbol{y} \mid f)$ is Gaussian, the combination with a GP prior $\mathrm{P}(f \mid \boldsymbol{x})$ gives a posterior GP over functions. In binary classification, the distribution over the latent $f_{*}$ is combined with the logistic function to produce the prediction:

$$
\bar{\pi}_{*}=\int \sigma\left(f_{*}\right) \mathrm{P}\left(f_{*} \mid \boldsymbol{x}, \boldsymbol{y}, x_{*}\right) d f_{*} .
$$

This results in a non-Gaussian likelihood in the posterior formulation and therefore, exact inference is infeasible for classification models. Multiple approximations exist that make the computation tractable (Gibbs and Mackay, 1997; Williams and Barber, 1998; Neal, 1999). In our experiments we opt to use the Expectation Propagation (EP) method (Minka, 2001) which approximates the nonGaussian joint posterior with a Gaussian one. EP offers very good empirical results for many different likelihoods, although it has no proof of convergence. The complexity for the inference step is $\mathcal{O}\left(n^{3}\right)$. Given that our data set is very large and the number of features is high, we conduct inference using the fully independent training conditional (FITC) approximation (Snelson and Ghahramani, 2006) with 500 random inducing points. We refer the interested reader to Rasmussen and Williams (2006) for further information on GP classification.

Although we could use multi-class classification methods, in order to provide insight, we perform a separate one-vs-all classification for each class and then determine a label through the occupational class that has the highest likelihood.

\section{Experiments}

This section presents the experimental results for our task. We first compare the accuracy of our classification methods on held out data using each feature set and conduct a standard error analysis. We then use the interpretability of the ARD lengthscales from the GP classifier to further analyse the relevant features.

\subsection{Predictive Accuracy}

We assign users to one of nine possible classes (see the 'Major Groups' on Table 1) using one set of

\begin{tabular}{|l|c|c|c|}
\hline Feature & LR & SVM & GP \\
\hline Most frequent class & $34.4 \%$ & $34.4 \%$ & $34.4 \%$ \\
UserLevel & $34.0 \%$ & $31.5 \%$ & $34.2 \%$ \\
SVD-E-30 & $36.3 \%$ & $35.0 \%$ & $39.8 \%$ \\
SVD-E-50 & $36.7 \%$ & $36.9 \%$ & $38.6 \%$ \\
SVD-E-100 & $40.8 \%$ & $41.9 \%$ & $40.9 \%$ \\
SVD-E-200 & $40.0 \%$ & $43.1 \%$ & $43.8 \%$ \\
SVD-C-30 & $36.9 \%$ & $36.5 \%$ & $38.2 \%$ \\
SVD-C-50 & $37.7 \%$ & $38.3 \%$ & $40.5 \%$ \\
SVD-C-100 & $40.4 \%$ & $42.1 \%$ & $44.6 \%$ \\
SVD-C-200 & $44.2 \%$ & $47.9 \%$ & $48.2 \%$ \\
W2V-E-50 & $42.5 \%$ & $49.0 \%$ & $48.4 \%$ \\
W2V-C-30 & $40.0 \%$ & $46.0 \%$ & $47.1 \%$ \\
W2V-C-50 & $42.3 \%$ & $48.5 \%$ & $47.9 \%$ \\
W2V-C-100 & $44.4 \%$ & $48.7 \%$ & $51.3 \%$ \\
W2V-C-200 & $\mathbf{4 6 . 9 \%}$ & $\mathbf{5 1 . 7 \%}$ & $\mathbf{5 2 . 7 \%}$ \\
\hline
\end{tabular}

Table 3: 9-way classification accuracy on held-out data for our 3 methods. Textual features are obtained using SVD or Word2Vec (W2V). E represents embeddings, $\mathbf{C}$ clusters. The final number denotes the amount of clusters or the size of the embedding.

features at a time. Experiments combining features yielded only minor improvements. We apply common linear and non-linear methods together with our proposed GP classifier. The linear method is logistic regression (LR) with Elastic Net regularisation (Freedman, 2009) and the non-linear one is formulated by a Support Vector Machine (SVM) with an RBF kernel (Vapnik, 1998). The accuracy of our classifiers is measured on held-out data. Our data set is divided into stratified training $(80 \%)$, validation (10\%) and testing (10\%) sets. The validation set was used to learn the LR and SVM hyperparameters, while the GP did not use this set at all. We report results using all three methods and all feature sets in Table 3.

We first observe that user level features (UserLevel; see Section 4.1) are not useful for predicting the job class. This finding indicates that general social behaviour or user impact are likely to be spread evenly across classes. It also highlights the difficulty of the task and motivates the use of deeper textual features.

The textual features (see Section 4.2) improve performance as compared to the most frequent class baseline. We also notice that the embeddings (SVD$\mathrm{E}$ and W2V-E) have lower performance than the clusters (SVD-C and W2V-C) in most of the cases. This is expected, as adding word vectors to represent a user's text may overemphasise common words. The size of the embedding also increases performance. The $\mathrm{W} 2 \mathrm{~V}$ features show better ac- 


\begin{tabular}{|c|c|c|c|c|c|}
\hline Rank & Topic \# & Label & Topic (most central words; most frequent words) & MRR & $\mu(l)$ \\
\hline 1 & 116 & Arts & $\begin{array}{c}\text { archival, stencil, canvas, minimalist, illustration, paintings, abstract, designs, } \\
\text { lettering, steampunk; art, design, print, collection, poster, painting, custom, logo, } \\
\text { printing, drawing }\end{array}$ & .43 & 1.35 \\
\hline 2 & 105 & Health & $\begin{array}{c}\text { chemotherapy, diagnosis, disease, inflammation, diseases, arthritis, symptoms, } \\
\text { patients, mrsa, colitis; risk, cancer, mental, stress, patients, treatment, surgery, } \\
\text { disease, drugs, doctor }\end{array}$ & .20 & 2.76 \\
\hline 3 & 153 & Beauty Care & $\begin{array}{l}\text { exfoliating, cleanser, hydrating, moisturizer, moisturiser, shampoo, lotions, } \\
\text { serum, moisture, clarins; beauty, natural, dry, skin, massage, plastic, spray, } \\
\text { facial, treatments, soap }\end{array}$ & .19 & 3.69 \\
\hline 4 & 21 & $\begin{array}{l}\text { Higher } \\
\text { Education }\end{array}$ & $\begin{array}{c}\text { undergraduate, doctoral, academic, students, curriculum, postgraduate, enrolled, } \\
\text { master's, admissions, literacy; students, research, board, student, college, } \\
\text { education, library, schools, teaching, teachers }\end{array}$ & .18 & 3.21 \\
\hline 5 & 158 & $\begin{array}{c}\text { Software } \\
\text { Engineering }\end{array}$ & $\begin{array}{c}\text { integrated, data, implementation, integration, enterprise, configuration, } \\
\text { open-source, cisco, proprietary, avaya; service, data, system, services, access, } \\
\text { security, development, software, testing, standard }\end{array}$ & .17 & 3.10 \\
\hline 7 & 186 & Football & $\begin{array}{c}\text { bardsley, etherington, gallas, heitinga, assou-ekotto, lescott, pienaar, warnock, } \\
\text { ridgewell, jenas; van, foster, cole, winger, terry, reckons, youngster, rooney, } \\
\text { fielding, kenny }\end{array}$ & .16 & 3.11 \\
\hline 8 & 124 & Corporate & $\begin{array}{c}\text { consortium, institutional, firm's, acquisition, enterprises, subsidiary, corp, } \\
\text { telecommunications, infrastructure, partnership; patent, industry, reports, global, } \\
\text { survey, leading, firm, 2015, innovation, financial }\end{array}$ & .15 & 2.44 \\
\hline 9 & 96 & Cooking & $\begin{array}{l}\text { parmesan, curried, marinated, zucchini, roasted, coleslaw, salad, tomato, spinach, } \\
\text { lentils; recipe, meat, salad, egg, soup, sauce, beef, served, pork, rice }\end{array}$ & .15 & 3.00 \\
\hline 12 & 164 & $\begin{array}{l}\text { Elongated } \\
\text { Words }\end{array}$ & $\begin{array}{l}\text { yaaayy, wooooo, woooo, yayyyyy, yaaaaay, yayayaya, yayy, yaaaaaaay, } \\
\text { wooohooo, yaayyy; wait, till, til, yay, ahhh, hoo, woo, woot, whoop, woohoo }\end{array}$ & .11 & 3.47 \\
\hline 16 & 176 & Politics & $\begin{array}{c}\text { religious, colonialism, christianity, judaism, persecution, fascism, marxism, } \\
\text { nationalism, communism, apartheid; human, culture, justice, religion, democracy, } \\
\text { religious, humanity, tradition, ancient, racism }\end{array} \mid$ & .08 & 3.09 \\
\hline
\end{tabular}

Table 4: Topics, represented by their most central and most frequent 10 words, sorted by their ARD lengthscale MRR across the nine GP-based occupation classifiers. $\mu(l)$ denotes the average lengthscale for a topic across these classifiers. Topic labels are manually created.

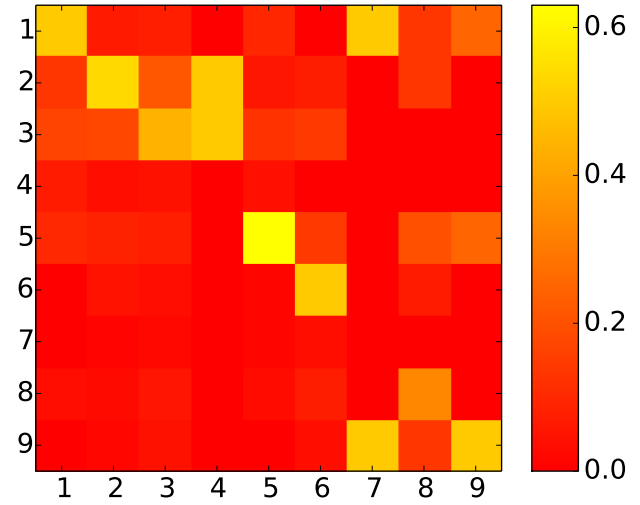

Figure 1: Confusion matrix of the prediction results. Rows represent the actual occupational class $(\mathbf{C} 1-$ 9) and columns the predicted class.

curacy than the SVD on the NPMI matrix. This is consistent with previous work that showed the efficiency of word2vec and the ability of those embeddings to capture non-linear relationships and syntactic features (Mikolov et al., 2013a; Mikolov et al., 2013b; Mikolov et al., 2013c).

LR has a lower performance than the non-linear methods, especially when using clusters as features. GPs usually outperform SVMs by a small margin. However, these offer the advantages of not using the validation set and the interpretability properties we highlight in the next section. Although we only draw our focus on major occupational classes, the data set allows the study of finer granularities of occupation classes in future work. For example, prediction performance for sub-major groups reaches $33.9 \%$ accuracy (15.6\% majority class, 22 classes) and $29.2 \%$ accuracy for minor groups (3.4\% majority class, 55 classes).

\subsection{Error Analysis}

To illustrate the errors made by our classifiers, Figure 1 shows the confusion matrix of the classification results. First, we observe that class 4 is many times classified as class 2 or 3 . This can be explained by the fact that classes 2,3 and 4 contain similar types of occupations, e.g. doctors and nurses or accountants and assistant accountants. However, with very few exceptions, we notice that only adjacent classes get misclassified, suggesting 
that our model captures the general user skill level.

\subsection{Qualitative Analysis}

The word clusters that were built from a reference corpus and then used as features in the GP classifier, give us the opportunity to extract some qualitative derivations from our predictive task. For the rest of the section we use the best performing model of this type (W2V-C-200) in order to analyse the results. Our main assumption is that there might be a divergence of language and topic usage across occupational classes following previous studies in sociology (Bernstein, 1960; Bernstein, 2003). Knowing that the inferred GP lengthscale hyperparameters are inversely proportional to feature (i.e. topic) relevance (see Section 5), we can use them to rank the topic importance and give answers to our hypothesis.

Table 4 shows 10 of the most informative topics (represented by the top 10 most central and frequent words) sorted by their ARD lengthscale Mean Reciprocal Rank (MRR) (Manning et al., 2008) across the nine classifiers. Evidently, they cover a broad range of thematic subjects, including potentially work specific topics in different domains such as 'Corporate' (Topic \#124), 'Software Engineering' (\#158), 'Health' (\#105), 'Higher Education' (\#21) and 'Arts' (\#116), as well as topics covering recreational interests such as 'Football' (\#186), 'Cooking' (\#96) and 'Beauty Care' (\#153).

The highest ranked MRR GP lengthscales only highlight the topics that are the most discriminative of the particular learning task, i.e. which topic used alone would have had the best performance. To examine the difference in topic usage across occupations, we illustrate how six topics are covered by the users of each class. Figure 2 shows the Cumulative Distribution Functions (CDFs) across the nine different occupational classes for these six topics. CDFs indicate the fraction of users having at least a certain topic proportion in their tweets. A topic is more prevalent in a class, if the CDF line leans towards the bottom-right corner of the plot.

'Higher Education' (\#21) is more prevalent in classes 1 and 2, but is also discriminative for classes 3 and 4 compared to the rest. This is expected because the vast majority of jobs in these classes require a university degree (holds for all of the jobs in classes 2 and 3) or are actually jobs in higher education. On the other hand, classes 5 to 9 have a similar behaviour, tweeting less on this topic. We also observe that words in 'Corporate' (\#124) are used more as the skill required for a job gets higher. This topic is mainly used by people in classes 1 and 2 and with less extent in classes 3 and 4, indicating that people in these occupational classes are more likely to use social media for discussions about corporate business.

There is a clear trend of people with more skilled jobs to talk about 'Politics' (\#176). Indeed, highly ranked politicians and political philosophers are parts of classes 1 and 2 respectively. Nevertheless, this pattern expands to the entire spectrum of the investigated occupational classes, providing further proof-of-concept for our methodology, under the assumption that the theme of politics is more attractive to the higher skilled classes rather than the lower skilled occupations. By examining 'Arts' (\#116), we see that it clearly separates class 5 , which includes artists, from all others. This topic appears to be relevant to most of the classification tasks and it is ranked first according to the MRR metric. Moreover, we observe that people with higher skilled jobs and education (classes 1-3) post more content about arts. Finally, we examine two topics containing words that can be used in more informal occasions, i.e. 'Elongated Words' (\#164) and 'Beauty Care' (\#153). We observe a similar pattern in both topics by which users with lower skilled jobs tweet more often.

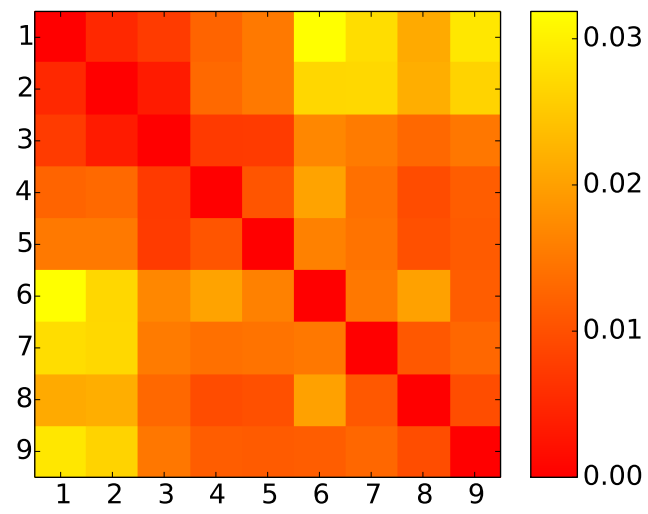

Figure 3: Jensen-Shannon divergence in the topic distributions between the different occupational classes (C 1-9).

The main conclusion we draw from Figure 2 is that there exists a topic divergence between users in the lower vs. higher skilled occupational classes. To examine this distinction better, we use the JensenShannon divergence (JSD) to quantify the difference between the topic distributions across every 

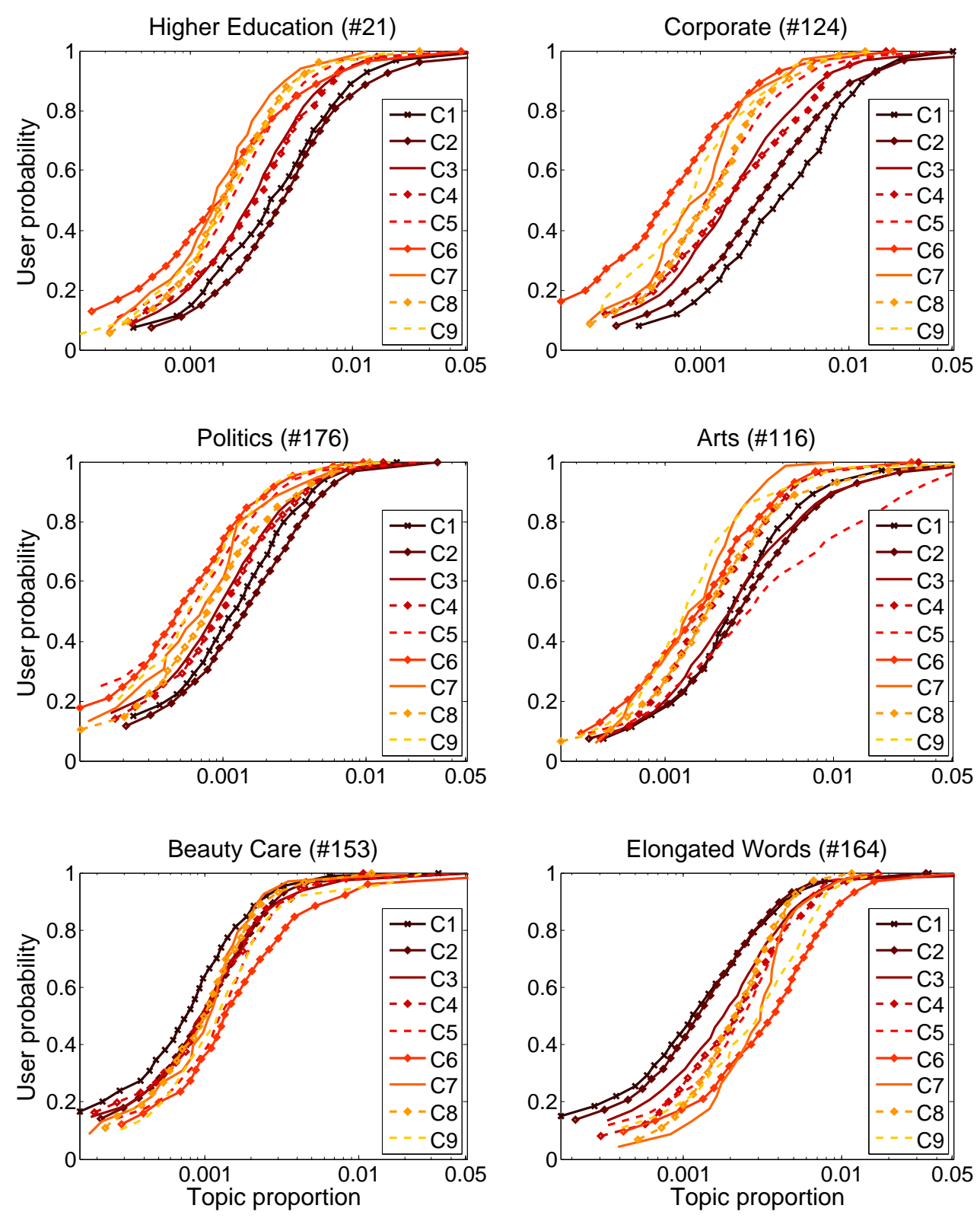

Figure 2: CDFs for six of the most important topics; the $\mathrm{x}$-axis is on the log-scale for display purposes. A point on a CDF line indicates the fraction of users ( $y$-axis point) with a topic proportion in their tweets lower or equal to the corresponding $\mathrm{x}$-axis point. The topic is more prevalent in a class, if the CDF line leans closer to the bottom-right corner of the plot.

class pair. Figure 3 visualises these differences. There, we confirm that adjacent classes use similar topics of discussion. We also notice that JSD increases as the classes are further apart. Two main groups of related classes, with a clear separation from the rest, are identified: classes 1-2 and 6-9. For the users belonging to these two groups, we compute their topic usage distribution (for the top topics listed in Table 4). Then, we assess whether the topic usage distributions of those super-classes of occupations have a statistically significant dif- ference by performing a two-sample KolmogorovSmirnov test. We enumerate the group topic usage means in Table 5; all differences were indeed statistically significant $\left(p<10^{-5}\right)$. From this comparison, we conclude that users in the higher skilled classes have a higher representation in all top topics but 'Beauty Care' and 'Elongated Words'. Hence, the original hypothesis about the difference in the usage of language between upper and lower occupational classes is reconfirmed in this more generic testing. A very noticeable difference occurs for the 


\begin{tabular}{l|c|c} 
Topics & C 1-2 & C 6-9 \\
\hline Arts & 4.95 & 2.79 \\
Health & 4.45 & 2.13 \\
Beauty Care & 1.40 & 2.24 \\
Higher Education & 6.04 & 2.56 \\
Software Engineering & 6.31 & 2.54 \\
Football & 0.54 & 0.52 \\
Corporate & 5.15 & 1.41 \\
Cooking & 2.81 & 2.49 \\
Elongated Words & 1.90 & 3.78 \\
Politics & 2.14 & 1.06 \\
\hline
\end{tabular}

Table 5: Comparison of mean topic usage for super-sets (classes 1-2 vs. 6-9) of the occupational classes; all values were multiplied by $10^{3}$. The difference between the topic usage distributions was statistically significant $\left(p<10^{-5}\right)$.

'Corporate' topic, whereas 'Football' registers the lowest distance.

\section{Related Work}

Occupational class prediction has been studied in the past in the areas of psychology and economics. French (1959) investigated the relation between various measures on 232 undergraduate students and their future occupations. This study concluded that occupational membership can be predicted from variables such as the ability of subjects in using mathematical and verbal symbols, their family economic status, body-build and personality components. Schmidt and Strauss (1975) also studied the relationship between job types (five classes) and certain demographic attributes (gender, race, experience, education, location). Their analysis identified biases or discrimination which possibly exist in different types of jobs. Sociolinguistic and sociology studies deduct that social status is an important factor in determining the use of language (Bernstein, 1960; Bernstein, 2003; Labov, 2006). Differences arise either due to language use or due to the topics people discuss as parts of various social domains. However, a large scale investigation of this hypothesis has never been attempted.

Relevant to our task is a relation extraction approach proposed by Li et al. (2014) aiming to extract user profile information on Twitter. They used a weakly supervised approach to obtain information for job, education and spouse. Nonetheless, the information relevant to the job attribute re- gards the employer of a user (i.e. the name of a company) rather than the type of occupation. In addition, Huang et al. (2014) proposed a method to classify Sina Weibo users to twelve predefined occupations using content based and network features. However, there exist significant differences from our task since this inference is based on a distinct platform, with an ambiguous distribution over occupations (e.g. more than $25 \%$ related to media), while the occupational classes are not generic (e.g. media, welfare and electronic are three of the twelve categories). Most importantly, the applied model did not allow for a qualitative interpretation. Filho et al. (2014) inferred the social class of social media users by combining geolocation information derived from Foursquare and Twitter posts. Recently, Sloan et al. (2015) introduced tools for the automated extraction of demographic data (age, occupation and social class) from the profile descriptions of Twitter users using a similar method to our data set extraction approach. They showed that it is feasible to build a data set that matches the real-world UK occupation distribution as given by the SOC.

\section{Conclusions}

Our paper presents the first large-scale systematic study on language use on social media as a factor for inferring a user's occupational class. To address this problem, we have also introduced an extensive labelled data set extracted from Twitter. We have framed prediction as a classification task and, to this end, we used the powerful, non-linear GP framework that combines strong predictive performance with feature interpretability. Results show that we can achieve a good predictive accuracy, highlighting that the occupation of a user influences text use. Through a qualitative analysis, we have shown that the derived topics capture both occupation specific interests as well as general class-based behaviours. We acknowledge that the derivations of this study, similarly to other studies in the field, are reflecting the Twitter population and may experience a bias introduced by users self-mentioning their occupations. However, the magnitude, occupational diversity and face validity of our conclusions suggest that the presented approach is useful for future downstream applications. 


\section{Acknowledgements}

DP-P acknowledges the support from Templeton Religion Trust, grant TRT-0048. VL and NA acknowledge the support from EPSRC (UK) project EP/K031953/1. We thank Mark Stevenson for his critical comments on early drafts of this paper.

\section{References}

Faiyaz Al Zamal, Wendy Liu, and Derek Ruths. 2012. Homophily and Latent Attribute Inference: Inferring Latent Attributes of Twitter Users from Neighbors. In Proc. of 6th International Conference on Weblogs and Social Media, pages 387-390.

Basil Bernstein. 1960. Language and social class. British Journal of Sociology, pages 271-276.

Basil Bernstein. 2003. Class, codes and control: Applied studies towards a sociology of language, volume 2. Psychology Press.

Johan Bollen, Huina Mao, and Xiaojun Zeng. 2011. Twitter mood predicts the stock market. Journal of Computational Science, 2(1):1-8.

Gerlof Bouma. 2009. Normalized (pointwise) mutual information in collocation extraction. In Biennial GSCL Conference, pages 31-40.

D. John Burger, John Henderson, George Kim, and Guido Zarrella. 2011. Discriminating Gender on Twitter. In Proceedings of the 2011 Conference on Empirical Methods in Natural Language Processing, EMNLP, pages 1301-1309.

Zhiyuan Cheng, James Caverlee, and Kyumin Lee. 2010. You are where you tweet: a content-based approach to geo-locating twitter users. In Proceedings of the 19th ACM Conference on Information and Knowledge Management, CIKM, pages 759-768.

Trevor Cohn and Lucia Specia. 2013. Modelling annotator bias with multi-task gaussian processes: An application to machine translation quality estimation. In 51st Annual Meeting of the Association for Computational Linguistics, ACL, pages 32-42.

Glen Coppersmith, Craig Harman, and Mark Dredze. 2014. Measuring post traumatic stress disorder in twitter. In International Conference on Weblogs and Social Media, ICWSM.

Renato Miranda Filho, Guilherme R. Borges, Jussara M. Almeida, and Gisele L. Pappa. 2014. Inferring user social class in online social networks. In Proceedings of the 8th Workshop on Social Network Mining and Analysis, SNAKDD'14, pages 10:110:5.

David Freedman. 2009. Statistical models: theory and practice. Cambridge University Press.
Wendell L French. 1959. Can a man's occupation be predicted? Journal of Counseling Psychology, 6(2):95.

Mark Gibbs and David J. C. Mackay. 1997. Variational gaussian process classifiers. IEEE Transactions on Neural Networks, 11:1458-1464.

Brent Hecht, Lichan Hong, Bongwon Suh, and Ed H. Chi. 2011. Tweets from justin bieber's heart: The dynamics of the location field in user profiles. In Proceedings of the SIGCHI Conference on Human Factors in Computing Systems, CHI.

Yanxiang Huang, Lele Yu, Xiang Wang, and Bin Cui. 2014. A multi-source integration framework for user occupation inference in social media systems. World Wide Web, pages 1-21.

William Labov. 2006. The Social Stratification of English in New York City. Cambridge University Press, second edition.

Vasileios Lampos and Nello Cristianini. 2010. Tracking the flu pandemic by monitoring the Social Web. In Proc. of the 2nd International Workshop on Cognitive Information Processing, pages 411-416.

Vasileios Lampos and Nello Cristianini. 2012. Nowcasting Events from the Social Web with Statistical Learning. ACM Transactions on Intelligent Systems and Technology, 3(4):72:1-72:22.

Vasileios Lampos, Daniel Preoţiuc-Pietro, and Trevor Cohn. 2013. A user-centric model of voting intention from Social Media. In Proceedings of the 51st Annual Meeting of the Association for Computational Linguistics, ACL, pages 993-1003.

Vasileios Lampos, Nikolaos Aletras, Daniel PreoțiucPietro, and Trevor Cohn. 2014. Predicting and characterising user impact on Twitter. In Proceedings of the 14th Conference of the European Chapter of the Association for Computational Linguistics, EACL, pages 405-413.

Omer Levy and Yoav Goldberg. 2014. Neural word embeddings as implicit matrix factorization. In $\mathrm{Ad}$ vances in Neural Information Processing Systems, NIPS, pages 2177-2185.

Jiwei Li, Alan Ritter, and Eduard H. Hovy. 2014. Weakly supervised user profile extraction from twitter. In Proceedings of the 52nd Annual Meeting of the Association for Computational Linguistics, ACL, pages 165-174.

Christopher D. Manning, Prabhakar Raghavan, and Hinrich Schütze. 2008. Introduction to Information Retrieval. Cambridge University Press.

Tomas Mikolov, Kai Chen, Greg Corrado, and Jeffrey Dean. 2013a. Efficient estimation of word representations in vector space. In Proceedings of Workshop at the International Conference on Learning Representations, ICLR. 
Tomas Mikolov, Ilya Sutskever, Kai Chen, Greg Corrado, and Jeffrey Dean. 2013b. Distributed representations of words and phrases and their compositionality. In Advances in Neural Information Processing Systems, NIPS, pages 3111-3119.

Tomas Mikolov, Wen tau Yih, and Geoffrey Zweig. 2013c. Linguistic regularities in continuous space word representations. In Proceedings of the 2010 annual Conference of the North American Chapter of the Association for Computational Linguistics, NAACL, pages 746-751.

Thomas P. Minka. 2001. Expectation propagation for approximate bayesian inference. In Proceedings of the 17th Conference in Uncertainty in Artificial Intelligence, UAI ' 01 .

Radford M. Neal. 1996. Bayesian Learning for Neural Networks. Springer-Verlag New York, Inc.

Radford M. Neal. 1999. Regression and classification using gaussian process priors. Bayesian Statistics 6, pages $475-501$.

Andrew Y. Ng, Michael I. Jordan, and Yair Weiss 2002. On spectral clustering: Analysis and an algorithm. In Advances in Neural Information Processing Systems, NIPS, pages 849-856.

Marco Pennacchiotti and Ana-Maria Popescu. 2011. A machine learning approach to twitter user classification. ICWSM, pages 281-288.

Tamara Polajnar, Simon Rogers, and Mark Girolami. 2011. Protein interaction detection in sentences via gaussian processes; a preliminary evaluation. International Journal of Data Mining and Bioinformatics, 5(1):52-72.

Daniel Preoţiuc-Pietro and Trevor Cohn. 2013. A temporal model of text periodicities using Gaussian Processes. EMNLP.

Daniel Preoţiuc-Pietro, Sina Samangooei, Trevor Cohn, Nicholas Gibbins, and Mahesan Niranjan. 2012. Trendminer: An architecture for real time analysis of social media text. In Workshop on Real-Time Analysis and Mining of Social Streams (RAMSS), ICWSM.

Delip Rao, David Yarowsky, Abhishek Shreevats, and Manaswi Gupta. 2010. Classifying Latent User Attributes in Twitter. In Proceedings of the 2nd International Workshop on Search and Mining Usergenerated Contents, SMUC, pages 37-44.

Carl Edward Rasmussen and Christopher K. I. Williams. 2006. Gaussian Processes for Machine Learning. The MIT Press.

Adam Sadilek, Henry Kautz, and Vincent Silenzio. 2012. Modeling Spread of Disease from Social Interactions. In Proc. of 6th International Conference on Weblogs and Social Media, pages 322-329.
Peter Schmidt and Robert P Strauss. 1975. The prediction of occupation using multiple logit models. International Economic Review, 16(2):471-86.

Jianbo Shi and Jitendra Malik. 2000. Normalized cuts and image segmentation. Transactions on Pattern Analysis and Machine Intelligence, 22(8):888-905.

Luke Sloan, Jeffrey Morgan, Pete Burnap, and Matthew Williams. 2015. Who tweets? Deriving the demographic characteristics of age, occupation and social class from twitter user meta-data. PloS one, 10(3):e0115545

Edward Snelson and Zoubin Ghahramani. 2006. Sparse gaussian processes using pseudo-inputs. In Advances in Neural Information Processing Systems, NIPS, pages 1257-1264.

Andranik Tumasjan, Timm Oliver Sprenger, Philipp G Sandner, and Isabell M Welpe. 2010. Predicting Elections with Twitter: What 140 Characters Reveal about Political Sentiment. In Proc. of 4th International Conference on Weblogs and Social Media, pages $178-185$.

Vladimir N Vapnik. 1998. Statistical learning theory. Wiley, New York.

Ulrike von Luxburg. 2007. A tutorial on spectral clustering. Statistics and computing, 17(4):395-416.

Christopher K.I Williams and David Barber. 1998. Bayesian classification with gaussian processes. IEEE Transactions on Pattern Analysis and Machine Intelligence, 20 (12):1342-1351. 